Environmetrics 2016; 27: 439-450

\title{
Bayesian inference for non-stationary marginal extremes
}

\author{
D. Randell, K. Turnbull, K. Ewans and P. Jonathan \\ (First revision, accepted by Environmetrics June 2016)
}

We propose a simple piecewise model for a sample of peaks-over-threshold, non-stationary with respect to multidimensional covariates, estimated using a carefully-designed computationally-efficient Bayesian inference. Model parameters are themselves parameterised as functions of covariates using penalised B-spline representations. This allows detailed characterisation of non-stationarity extreme environments. The approach gives similar inferences to a comparable frequentist penalised maximum likelihood method, but is computationally considerably more efficient and allows a more complete characterisation of uncertainty in a single modelling step. We use the model to quantify the joint directional and seasonal variation of storm peak significant wave height at a northern North Sea location, and estimate predictive directional-seasonal return value distributions necessary for the design and reliability assessment of marine and coastal structures.

\section{Introduction}

Bayesian inference provides an intuitive framework for environmental applications of extreme value analysis, allowing e.g. incorporation of prior knowledge and thorough uncertainty quantification within a single inference step, predictive inference, and when carefully designed a computationally efficient inference. Coles and Powell (1996) and Stephenson (2016) provide useful reviews. There are many applications of Bayesian inference for extreme value models in the literature. For example, Coles and Tawn (1996) reports a Bayesian analysis of extreme rainfall data. Coles and Tawn (2005) estimates extreme surges on the UK east coast for improved flood risk assessment, Cooley et al. (2006) explores Bayesian extremes in lichenometry as a means of understanding climate change, Scotto and Guedes-Soares (2007) estimates the distributions of significant wave height and Mendes et al. (2010) considers extremes of wild-fires. Beirlant et al. (2004) and Northrop et al. (2015) consider the specification of objective priors for extreme value model parameters, and Smith and Walshaw (2003) of informative priors. Davison et al. (2012) consider Bayesian hierarchical models for spatial extremes.

Extreme value models describe the tails of distributions; using a sample of peaks over threshold, only the largest values are generally used in extreme value estimation. Choice of extreme value threshold is therefore generally critical (e.g. Scarrott and MacDonald 2012). For descriptions of whole samples, mixture models and piecewise distributions are attractive. For example, Frigessi et al. (2002) uses a dynamic mixture model for fire loss data comprising a generalised Pareto (GP) tail and a Weibull body, and Behrens et al. (2004) considers a piecewise distribution comprised of a parametric form for the body of data and a GP tail. MacDonald et al. (2011) proposes a piecewise distribution in which a kernel density estimate for the body is used in conjunction with a GP tail. In oceanographic applications, many distributional forms have been proposed for wave height and significant wave height, motivated by observation and physical considerations. Forristall (1978), Haver (1985), Smith and Naylor (1987), Battjes and Groenendijk (2000), Ferreira and Guedes Soares (2000), Prevosto et al. (2000), Scotto and Guedes-Soares (2000) and Guedes-Soares and Scotto (2001) provide useful examples. The Weibull distribution is often used to model ocean current speed and wind speed. Monahan (2006) shows the Weibull distribution to be a fair approximation for surface wind speeds, and Chu (2008) uses the Weibull to model upper tropical Pacific current speeds. Ashkenazy and Gildor (2011) finds the Weibull distribution to be a good approximation of ocean current speeds and highlights the effect of covariates. Morgan et al. (2011) explores the performance of different distributions for wind speeds. 
Incorporating non-stationarity, or the effect of covariates, in extreme value analysis is also important (e.g. Davison and Smith 1990, Jonathan et al. 2008). Anderson et al. (2001) describes a seasonal model for return values of significant wave height. Covariate effects are considered in Coles and Walshaw (1994) for directional wind speeds, and in Mendez et al. (2008) for seasonal significant wave height. Exploration of temporal nonstationarity in extremes can also be found in Renard et al. (2006), Cheng et al. (2014) and Ortego Martinez et al. (2014). There are many ways to parameterise non-stationary effects in statistical models, including penalised B-splines. Ugarte et al. (2012) and Oumow et al. (2012) use this formulism to estimate smooth parameter variation, the latter within a mixture model. Bayesian penalised B-splines as described by Brezger and Lang (2006) are particularly useful for the current work.

A plethora of different MCMC algorithms (e.g. Gamerman and Lopes 2006) is available to sample from posterior distributions. Here, we adopt a combination of different sampling schemes to achieve good computational efficiency and chain mixing, including direct Gibbs sampling of full conditional distributions and MetropolisHastings (MH) within Gibbs. We find it advantageous in $\mathrm{MH}$ sampling from some full conditionals to exploit gradient and curvature information to improve the quality of proposals. Where possible, we employ the Riemann manifold Metropolis-adjusted Langevin algorithm (mMALA) as described by Girolami and Calderhead (2011) and Xifara et al. (2014). Estimation schemes which exploit gradient and curvature information for problematic functions such as the GP likelihood are recommended (e.g. Jones et al. 2016).

The objective of the current work is to develop a simple whole-sample model for independent observations given multidimensional covariates, incorporating the generalised Pareto form for exceedances of a non-stationary threshold. The model is needed to estimate distributions of return values corresponding to long return periods, for use in the design and reliability assessment of marine and coastal structures. A major consideration in the development of the methodology is ease-of-use and computational efficiency for full-scale oceanographic applications for sample sizes from $10^{3}$ to $10^{7}$ with at least a two-dimensional (e.g. directional-seasonal) covariate domain.

The article is arranged as follows. Section 2 provides a motivating application to modelling of storm-peak significant wave height events at a location in the northern North Sea, subject to directional and seasonal variability in the rate and magnitude of occurrence of events. Section 3 introduces a piecewise truncated Weibull - generalised Pareto model for the size of events (Section 3.1), and a Poisson process model for the rate of occurrence of events (Section 3.2). Model parameters are non-stationary with respect to covariates, parameterised in terms of the tensor product of B-spline bases for the directional-seasonal covariate domain (Section 3.3). The specification of prior distributions for model parameters is discussed in Section 3.4, and Bayesian inference in Section 3.5. Application to the northern North Sea data is outlined in Section 4. Discussion and conclusions, including comments on a comparative study at this location and in the South China Sea, are given in Section 5. The appendix describes key elements of the inference scheme in more detail.

\section{Motivating application}

Significant wave height measures the energy (or roughness) of the ocean surface, and can be defined as four times the standard deviation of the ocean surface elevation at a spatial location for a specified period of observation. The application sample is taken from the WAM hindcast of Reistad et al. 2011, which provides time-series of significant wave height, (dominant) wave direction and season (defined as day of the year, for a standardised year consisting of 360 days) for three hour sea-states for the period September 1957 to December 2012 at a northern North Sea location. (A hindcast is a physical model of the ocean environment, incorporating pressure field, wind field and wind-wave generation models in particular; the hindcast model is calibrated to observations of the environment from instrumented offshore facilities, moored buoys and satellite altimeters in the neighbourhood of the location for a period of time, typically decades.) Aarnes et al. (2012) and Breivik et al. (2013) study extreme value characteristics of storm severities from the hindcast. Extreme sea states in the North Sea are dominated by winter storms originating in the Atlantic Ocean and propagating eastwards across the northern part of the North Sea. Due to their proximity to the storms, sea states at northern North Sea locations are usually more 
intense than in the southern North Sea. Occasionally, the storms travel south-eastward and intrude into the southern North Sea producing large sea states. Directions of propagation of extreme seas vary considerably with location, depending on land shadows of the British Isles, Scandinavia, and the coast of mainland Europe, and fetches associated with the Atlantic Ocean, Norwegian Sea, and the North Sea itself. In the northern North Sea the main fetches are the Norwegian Sea to the North, the Atlantic Ocean to the west, and the North Sea to the south. Extreme sea states from the directions of Scandinavia to the east and the British Isles to the south-west are not possible. The shielding by these land masses is more effective for southern North Sea locations, resulting in a similar directional distribution but reduced wave heights by comparison with northern North Sea locations.

Storm peak significant wave height characteristics are isolated from the hindcast time-series using the procedure described in Ewans and Jonathan (2008), for a location offshore Norway at a latitude exceeding $62^{\circ}$. Contiguous intervals of significant wave height above a low peak-picking threshold are identified, each interval now assumed to correspond to a storm event. The peak-picking threshold corresponds to a directional-seasonal quantile of significant wave height with specified non-exceedance probability, estimated using quantile regression. The maximum of significant wave height during the storm interval is taken as the storm peak significant wave height $\left(H_{S}\right.$ for brevity in this work). The values of directional and seasonal covariates at the time of storm peak significant wave height are referred to as storm peak values of those variables. The resulting storm peak sample consists of 2941 values of $H_{S}$. With direction from which a storm travels expressed in degrees clockwise with respect to north, Figure 1 shows a map of the region and plots of $H_{S}$ (in metres) versus direction and season.

It can be seen that the land shadow of Norway (approximately the directional interval $\left.\left(45^{\circ}, 210^{\circ}\right)\right)$ has a considerable effect on the rate and size of occurrences with direction. In particular, there is a dramatic increase in both rate and size of occurrences with increasing direction at around $210^{\circ}$, corresponding to Atlantic storm events from the south-west able to pass the Norwegian headland. We therefore should expect considerable directional variability in model parameter estimates for the sample. In contrast, the magnitude of the rate of change of both rate and size of occurrences with respect to season is lower; this should also be reflected in model parameters. Winter storms (approximately from October to March) are more intense and numerous.

\section{Model}

\subsection{Size of exceedance}

The magnitude $y$ of $H_{S}$ peaks-over-threshold events is assumed to follow a non-stationary piecewise (or two part) distribution, the form of which is motivated by both physical and statistical considerations. Wave models derived from theory or estimated historically from measurements (Section 1) suggest that a suitably-parameterised Weibull distribution provides an adequate representation for the body of the distribution of individual wave heights, crest elevations and storm severities ( such as $H_{S}$ ). Moreover, asymptotic theory suggest that an extreme value model is required to describe the largest threshold exceedances. We therefore specify the piecewise model as follows.

For response $y$, non-exceedances of some threshold $\psi$ are assumed to be distributed according to a nonstationary three-parameter truncated Weibull (TW) distribution with scale $\alpha(>0)$, shape $\gamma(>0)$, pre-specified location $\zeta(<\psi)$, and support $[\zeta, \psi]$. Threshold exceedances of $\psi$ are assumed to be distributed according to a non-stationary generalised Pareto (GP) distribution with scale $\sigma(>0)$, shape $\xi(\in \mathbb{R})$ and support $\left(\psi, y^{+}\right)$ (where $y^{+}=\psi-\frac{\sigma}{\xi}$ for $\xi<0$ and $=\infty$ otherwise). Note that all of $\alpha, \gamma, \sigma, \xi$ and $\zeta$ will be assumed to be smoothly-varying functions of direction and season in due course.

The corresponding probability density function

$$
f(y \mid \tau, \alpha, \gamma, \sigma, \xi)= \begin{cases}\tau \times f_{T W}(y \mid \tau, \alpha, \gamma) & \text { for } y \in[\zeta, \psi] \\ (1-\tau) \times f_{G P}(y \mid \tau, \alpha, \gamma, \sigma, \xi) & \text { for } y \in\left(\psi, y^{+}\right)\end{cases}
$$



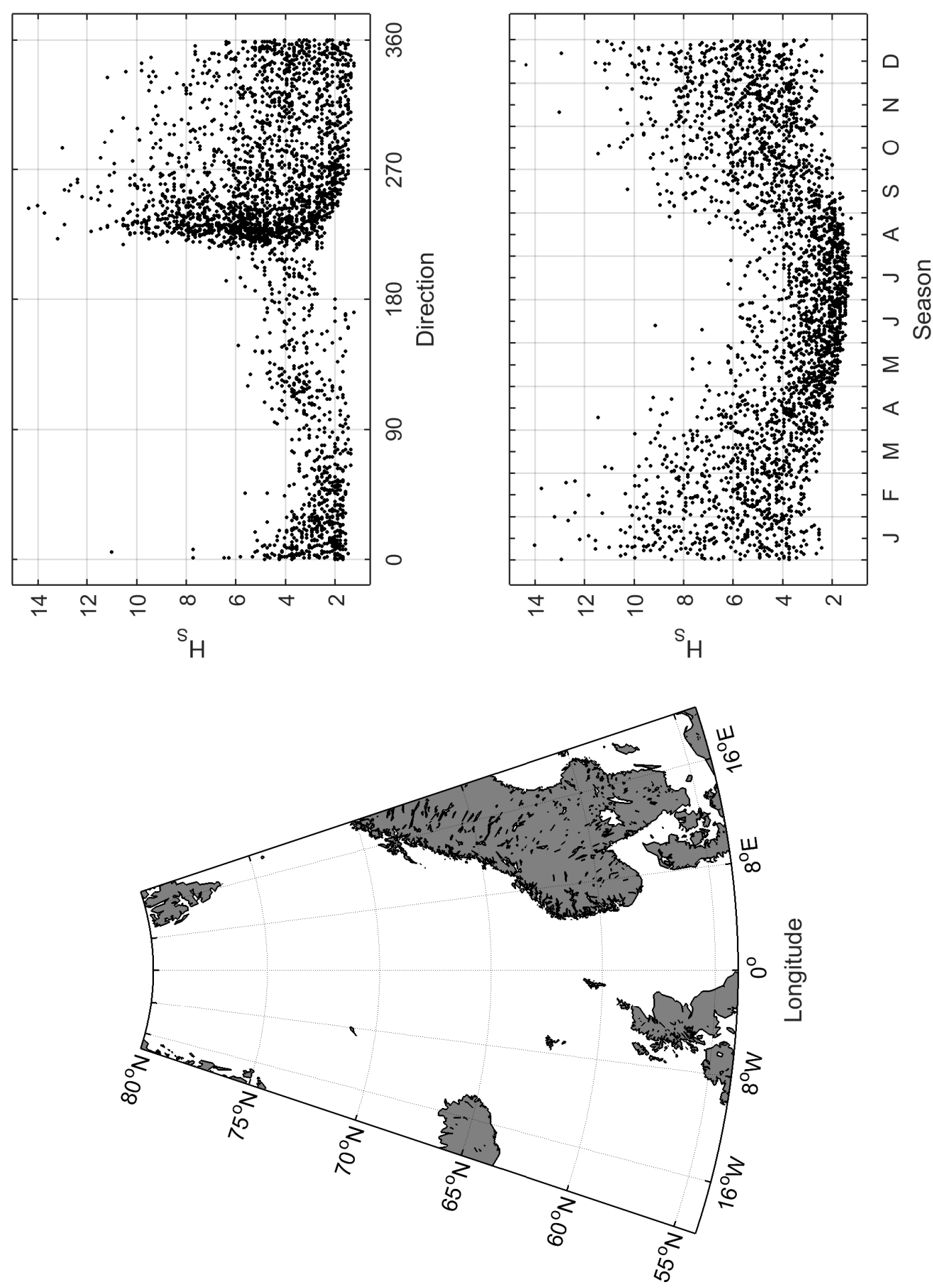

әрпฺ!ฺอา

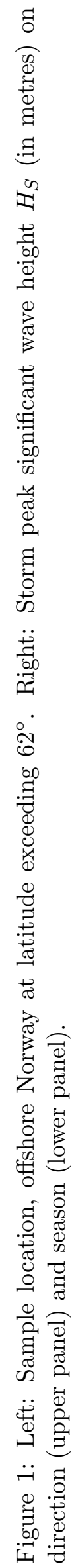


is similar to the (stationary) model of Behrens et al. (2004). The pre-specified location parameter $\zeta$ is omitted from this description since it is not inferred; in the current work, $\zeta$ corresponds to a non-stationary "peakpicking" threshold used for storm peak identification prior to model estimation (Section 2). The factors $\tau$ and $(1-\tau)$ in the piecewise density serve to weight the relative contributions of the TW and GP parts; parameter $\tau$ corresponds to the extreme value threshold non-exceedance probability assumed stationary with respect to covariates, providing a scalar transition to describe the transition from TW to GP. $\tau$ is estimated as part of the inference.

Below extreme value threshold $\psi, y$ follows a truncated Weibull distribution with density

$$
f_{T W}(y \mid \tau, \alpha, \gamma)=\frac{f_{W}(y \mid \alpha, \gamma)}{F_{W}(\psi \mid \alpha, \gamma, \tau)} \text { for } y \in[\zeta, \psi]
$$

where

$$
f_{W}(y \mid \alpha, \gamma)=\frac{\gamma}{\alpha}\left(\frac{y-\zeta}{\alpha}\right)^{\gamma-1} \exp \left(-\left(\frac{y-\zeta}{\alpha}\right)^{\gamma}\right) .
$$

Note the threshold $\psi$ is specified as $\zeta+\alpha(-\log (1-\tau))^{-1 / \gamma}$ such that

$$
F_{W}(\psi \mid \alpha, \gamma, \tau)=1-\exp \left(-\left(\frac{\psi-\zeta}{\alpha}\right)^{\gamma}\right)=\tau \text { always. }
$$

Above extreme value threshold $\psi, y$ follows a generalised Pareto distribution with density

$$
f_{G P}(y \mid \tau, \alpha, \gamma, \sigma, \xi)=\frac{1}{\sigma}\left(1+\frac{\xi}{\sigma}(y-\psi)\right)^{-1 / \xi-1} \text { for } y \in\left(\psi, y^{+}\right) .
$$

In practice, we choose to carry out computations with $\nu=\sigma(1+\xi)$ in place of $\sigma$. This choice is motivated by fact that, with $\xi>-0.5$, maximum likelihood estimates for $\nu$ and $\xi$ are asymptotically independent, simplifying their joint estimation compared with joint estimation of $\sigma$ and $\xi$. Nevertheless, (derived) estimates for $\sigma$ will in general be reported and visualised below where possible for ease of interpretation.

\subsection{Rate of occurrence}

The rate of occurrence of all events (both exceedances and non-exceedances of threshold $\psi$ ) are assumed to follow a non-stationary Poisson process with rate $\rho$ varying with direction and season. For events on covariate domain $\mathcal{D}$ (Section 3.3) at locations $\boldsymbol{r}=\left\{\boldsymbol{r}_{i}\right\}_{i=1}^{n}$, the Poisson process likelihood is $f(\boldsymbol{r} \mid \rho)=\exp \left(-\int_{\mathcal{D}} \rho(\boldsymbol{r}) d \boldsymbol{r}\right) \prod_{i=1}^{n} \rho\left(\boldsymbol{r}_{j}\right)$. Following Chavez-Demoulin and Davison (2005), we approximate this integral by evaluating it on the index set of $m$ directional-seasonal sub-intervals of area $d$, assuming $d$ small enough so that $\rho$ is constant on each sub-interval. Then, for a vector of counts $\boldsymbol{c}=\left\{c_{k}\right\}_{k=1}^{m}$ of occurrences on the index set

$$
f(\boldsymbol{c} \mid \rho)=\exp \left(-d \sum_{k=1}^{m} \rho_{k}\right) \prod_{k=1}^{m} \rho_{k}^{c_{k}}
$$

where $\boldsymbol{\rho}=\left\{\rho_{k}\right\}_{k=1}^{m}$ is the corresponding Poisson count rate.

\subsection{Spline parameterisation}

Physical considerations suggest we should expect model parameters $\rho, \alpha, \gamma, \nu$ and $\xi$ to vary smoothly with respect to directional and seasonal covariates (whereas threshold non-exceedance probability $\tau$ is assumed stationary). For estimation, this can be achieved by expressing each parameter in terms of an appropriate basis for the domain $\mathcal{D}$ of covariates, where $\mathcal{D}=\mathcal{D}_{2} \times \mathcal{D}_{1} . \mathcal{D}_{1}=\mathcal{D}_{2}=[0,360)$ are the (marginal) domains of storm direction and season respectively under consideration. We calculate a $m_{1} \times p_{1}$ periodic marginal B-spline basis 
matrix $\boldsymbol{B}_{1}$ for an index set of $m_{1}=50$ directional locations, and a $m_{2} \times p_{2}$ periodic marginal B-spline basis matrix $\boldsymbol{B}_{2}$ for an index set of $m_{2}=50$ seasonal locations, yielding a total of $m\left(=m_{1} m_{2}\right)$ combinations of covariate values in the index set on $\mathcal{D}$. Following the work of Eilers and Marx and coauthors (e.g. Marx and Eilers 1998, Eilers et al. 2006 and citations thereof), we define the $m \times p$ basis matrix $\boldsymbol{B}$ (where $p=p_{1} p_{2}$ ) for $\mathcal{D}$ using the tensor product $\boldsymbol{B}=\boldsymbol{B}_{2} \otimes \boldsymbol{B}_{1}$. The value of any parameter vector $\boldsymbol{\eta}$ on the 2D index set can therefore be expressed as $\boldsymbol{B} \boldsymbol{\beta}_{\eta}$ for some $p \times 1$ vector $\boldsymbol{\beta}_{\eta}$ of spline parameters. Model estimation reduces to estimating appropriate sets of spline parameters for each $\eta \in\{\rho, \alpha, \gamma, \sigma, \xi\}$. Here, marginally for periodic directional and seasonal bases, we set $p_{1}=p_{2}=10$, although we also considered $p_{1}=p_{2}=20$ (and $\left.m_{1}=m_{2}=100\right)$ to confirm lack of sensitivity of inferences to this choice.

In any single covariate dimension $j(=1,2)$, the roughness $R_{\eta j}^{\circ}$ of any $p_{j} \times 1$ spline parameter vector $\boldsymbol{\beta}_{\eta j}^{\circ}$ can be evaluated on the index set of $m_{j}$ locations as $R_{\eta j}^{\circ}=\boldsymbol{\beta}_{\eta j}^{\circ}{ }^{\prime} \boldsymbol{P}_{\eta j}^{\circ} \boldsymbol{\beta}_{\eta j}^{\circ}$ where $\boldsymbol{P}_{\eta j}^{\circ}$ is a $p_{j} \times p_{j}$ penalty matrix of the form $P_{\eta j}^{\circ}=\boldsymbol{D}_{\eta j}^{\circ}{ }^{\prime} \boldsymbol{\Delta}_{\eta j}^{\circ} \boldsymbol{D}_{\eta j}^{\circ}$. $\boldsymbol{D}_{\eta j}^{\circ}$ is a $r_{j} \times p_{j}$ difference matrix, taken in this work to be a first order difference, such that $\boldsymbol{D}_{\eta j}^{\circ} \boldsymbol{\beta}_{\eta j}^{\circ}$ yields a $r_{j} \times 1$ vector of differences between values of consecutive elements of $\boldsymbol{\beta}_{\eta j}^{\circ}$. $\boldsymbol{\Delta}_{\eta j}^{\circ}$ is a $r_{j} \times r_{j}$ diagonal matrix with the $r_{j} \times 1$ vector of roughness penalties $\boldsymbol{\delta}_{\eta j}^{\circ}$ on its leading diagonal, $\boldsymbol{\Delta}_{\eta j}^{\circ}=\operatorname{diag}\left(\boldsymbol{\delta}_{\eta j}^{\circ}\right)$. In this work, since both directional and seasonal domains are periodic, we have $r_{j}=p_{j}, j=1,2$.

Spline roughness $R_{\eta}$ on $\mathcal{D}$ can be expressed in terms of marginal characteristics using tensor products. We extend the marginal difference matrices using $\boldsymbol{D}_{\eta 1}=\boldsymbol{I}_{2} \otimes \boldsymbol{D}_{\eta 1}^{\circ}$ and $\boldsymbol{D}_{\eta 2}=\boldsymbol{D}_{\eta 2}^{\circ} \otimes \boldsymbol{I}_{1}$, where $\boldsymbol{I}_{1}$ and $\boldsymbol{I}_{2}$ are respectively $p_{1} \times p_{1}$ and $p_{2} \times p_{2}$ identity matrices. The extended $p \times p$ penalty matrices become $\boldsymbol{P}_{\eta j}=$ $\boldsymbol{D}_{\eta j}{ }^{\prime} \boldsymbol{\Delta}_{\eta j} \boldsymbol{D}_{\eta j}, j=1,2 . \quad \boldsymbol{\Delta}_{\eta 1}$ is a $p_{2} r_{1} \times p_{2} r_{1}$ diagonal matrix, the leading diagonal of which is the vector of spline penalties $\boldsymbol{\delta}_{\eta 1} \cdot \boldsymbol{\Delta}_{\eta 2}$ is defined analogously. The overall spline roughness penalty matrix on $\mathcal{D}$ is $\boldsymbol{P}_{\eta}=\lambda_{\eta 1} \boldsymbol{P}_{\eta 1}+\lambda_{\eta 2} \boldsymbol{P}_{\eta 2}$ for spline roughness penalty coefficient vector $\boldsymbol{\lambda}_{\eta}=\left(\lambda_{\eta 1}, \lambda_{\eta 2}\right)$. $\boldsymbol{P}_{\boldsymbol{\eta}}$ is used as the prior precision matrix for spline coefficients $\boldsymbol{\beta}_{\eta}$. Random roughness penalties, as opposed to fixed penalties of unit size, promote better mixing of the MCMC chain (see Brezger and Lang 2006).

The computational burden of the matrix manipulations above can become restrictive as $m$ and $p$ increase. Fortunately, using generalised linear array methods (GLAM, Currie et al. 2006) we avoid the need ever to calculate the full $m \times p$ basis matrix $\boldsymbol{B}$, since $\boldsymbol{B} \boldsymbol{\beta}_{\eta}$ is equivalent (after rearrangement) to $\boldsymbol{B}_{\eta 1} \boldsymbol{\mathcal { M }}\left(\boldsymbol{\beta}_{\eta}\right) \boldsymbol{B}_{\eta 2}^{\prime}$, where $\mathcal{M}\left(\boldsymbol{\beta}_{\eta}\right)$ is a $p_{1} \times p_{2}$ rearrangement of the $p \times 1$ vector $\boldsymbol{\beta}_{\eta}$.

\subsection{Prior specification}

The following prior specification is made for $\tau$, the components of vectors $\boldsymbol{\delta}_{\eta 1}, \boldsymbol{\delta}_{\eta 2}$ and the scalars $\lambda_{\eta 1}, \lambda_{\eta 2}$ for $\eta \in\{\rho, \alpha, \gamma, \nu, \xi\}$. The extreme threshold non-exceedance probability $\tau$ follows beta distribution $B\left(a_{\tau}, b_{\tau}\right)$, with fixed parameters. This distributional form is suitable for the description of a probability since it has range $[0,1]$. The choice of parameters $a_{\tau}, b_{\tau}$ was motivated by the requirement to have sufficient sample to estimate the generalised Pareto parameters well (i.e. $\tau$ relatively small) but also ensure reasonable extreme value tail fit (i.e. $\tau$ relatively large). The Supplementary Material accompanying this article provides further discussion of this topic. The two components of positive-valued spline roughness penalty coefficient vector $\boldsymbol{\lambda}_{\eta}$ are assumed to be gamma-distributed $\Gamma\left(a_{\lambda}, b_{\lambda}\right)$ independently and identically. This choice of prior ensures that the corresponding full conditional distributions are available in closed form, enabling Gibbs sampling. The distribution of the spline roughness penalty vector $\boldsymbol{\delta}_{\eta}$ is not inferred; its positive-valued components are taken to follow gamma distribution $\Gamma\left(a_{\delta}, b_{\delta}\right)$ identically and independently. This distributional form, and the parameter values for $a_{\delta}, b_{\delta}$ were recommended by Brezger and Lang 2006 and adopted here following some confirmatory exploratory analysis. Further description of prior specification, including values for $a_{\tau}, b_{\tau}, a_{\delta}, b_{\delta}, a_{\lambda}$ and $b_{\lambda}$ is given in the Appendix.

In addition, the vector of spline parameters $\boldsymbol{\beta}_{\eta}$ corresponding to $\eta \in\{\rho, \alpha, \gamma, \nu, \xi\}$ is distributed with density proportional to $\exp \left(-\frac{1}{2} \boldsymbol{\beta}_{\eta}^{\prime} \boldsymbol{P}_{\boldsymbol{\eta}} \boldsymbol{\beta}_{\eta}\right)$ (Brezger and Lang 2006), where penalty matrix $\boldsymbol{P}_{\boldsymbol{\eta}}$ is a function of $\boldsymbol{\delta}_{\eta}$ and $\boldsymbol{\lambda}_{\eta}$ (Section 3.3). 


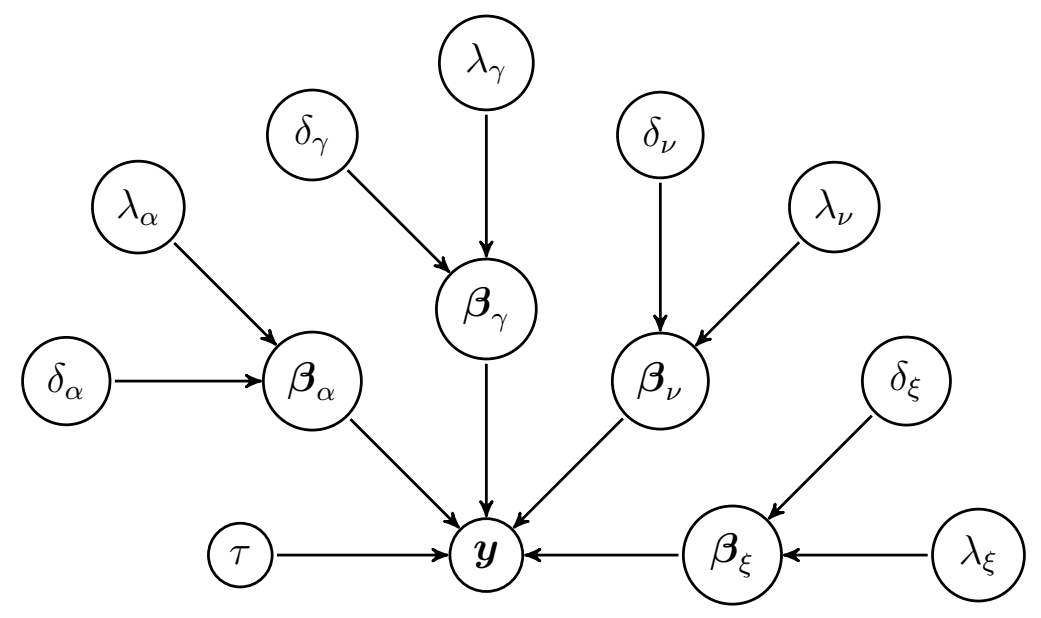

Figure 2: Directed acyclic graph for truncated Weibull - generalised Pareto inference of event magnitude.

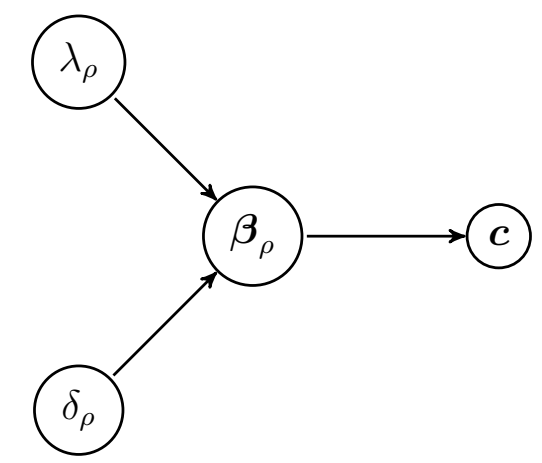

Figure 3: Directed acyclic graph for Poisson inference of event rate of occurrence.

\subsection{Inference}

For a sample $\boldsymbol{y}$ of event sizes, we estimate the model described in Section 3 with spline representations of the form $\boldsymbol{\eta}=\boldsymbol{B} \boldsymbol{\beta}_{\eta}$ on the index set of covariate locations, for parameters $\eta \in\{\rho, \alpha, \gamma, \nu, \xi\}$. The roughness of each $\eta$ with respect to directional and seasonal covariates respectively is regulated using the $2 \times 1$ roughness coefficient vector $\boldsymbol{\lambda}_{\eta}$ (itself inferred) and spline penalties $\boldsymbol{\delta}_{\eta}$ (drawn at random from an assumed known distribution). In addition, the non-exceedance probability $\tau$ corresponding to the extreme value threshold is estimated. The full parameter set is therefore $\Omega=\left\{\tau, \boldsymbol{\beta}_{\alpha}, \boldsymbol{\delta}_{\alpha}, \boldsymbol{\lambda}_{\alpha}, \boldsymbol{\beta}_{\gamma}, \boldsymbol{\delta}_{\gamma}, \boldsymbol{\lambda}_{\gamma}, \boldsymbol{\beta}_{\nu}, \boldsymbol{\delta}_{\nu}, \boldsymbol{\lambda}_{\nu}, \boldsymbol{\beta}_{\xi}, \boldsymbol{\delta}_{\xi}, \boldsymbol{\lambda}_{\xi}\right\}$. Inference can be expressed conveniently in terms of a directed acyclic graph (DAG). The analogous representation for a sample $\boldsymbol{c}$ of event rates is

The posterior distribution of model parameters is not available in closed form for either event size or rate of occurrence. Posterior inference is therefore made using Monte Carlo Markov chain (MCMC) by sampling from full conditionals as detailed in the Appendix and outlined below. When full conditionals are available in closed form, we use Gibbs sampling; otherwise, we use Metropolis-Hastings (MH) in Gibbs. When the function to be sampled is particularly problematic (e.g. posteriors for GP shape and adjusted scale), we exploit function gradient and curvature information if possible to generate $\mathrm{MH}$ proposals efficiently. In particular, full conditional distributions for the spline roughness penalty coefficients $\boldsymbol{\lambda}_{\eta}=\left(\lambda_{\eta 1}, \lambda_{\eta 2}\right)$ are available in closed form, permitting Gibbs sampling. With gamma prior specification (Section 3.4), the posterior distribution is $\Gamma\left(a_{\eta j}+\frac{1}{2} \operatorname{rank}\left(\boldsymbol{P}_{\eta}\right), b_{\eta j}+\frac{1}{2} \boldsymbol{\beta}_{\eta}^{\prime} \boldsymbol{P}_{\boldsymbol{\eta}} \boldsymbol{\beta}_{\eta}\right)$.

At each iteration of the MCMC chain, we sample in turn from the full conditional distribution of extreme 
threshold non-exceedance probability $\tau$, the spline parameters $\boldsymbol{\beta}_{\rho}$, then spline roughness penalties $\boldsymbol{\delta}_{\rho}$, then spline roughness penalty coefficients $\boldsymbol{\lambda}_{\rho}$ for Weibull scale $\alpha$. Then we sample from the corresponding triplets of parameters for TW shape $\alpha$ and shape $\gamma$, and GP shape $\xi$ and adjusted scale $\nu$. We experimented with different orderings of parameters and found there to be little difference in performance. Further algorithmic details are given in the Appendix.

\section{Application}

The model of Section 3 was applied to the northern North Sea application sample. The posterior distribution of parameters was estimated using MCMC, incorporating 20000 burn-in iterations, with the same number of subsequent iterations to estimate the posterior. Visual inspection of individual MCMC chains suggested good mixing; informal comparisons of multiple chains confirmed satisfactory convergence. Figure 4 shows directionalseasonal plots for posterior median TW, GP and Poisson rate parameter estimates, and prior (dashed red) and posterior densities for extreme value threshold non-exceedance probability $(\tau)$. The strong directional variability of rate of occurrence is most apparent, compared to more modest seasonal variation. TW and GP scale parameter estimates both indicate large event magnitudes at around $270^{\circ}$, corresponding to storms from the Atlantic. The GP shape parameter estimate is approximately -0.14, typical for northern North Sea locations. The posterior distribution of non-exceedance probability is relatively similar to its prior, implying that the sample provides relatively little evidence for the value of this parameter.

Figure 5 shows estimated posterior densities for directional and seasonal (dashed red) spline roughness penalty coefficients for TW, GP and Poisson rate parameters. Directional and seasonal densities for TW and GP shape parameters are very similar, suggesting that the same degree of spline roughness with respect to either covariate is appropriate. However, seasonal roughness penalties for TW and GP scale parameters are higher than directional roughness penalties, indicating that scale parameters show more directional variability.

Using the estimated posterior distribution $f(\Omega \mid \boldsymbol{y}, \boldsymbol{c})$ of parameters $\Omega$ given sample $\{\boldsymbol{y}, \boldsymbol{c}\}$, we can estimate predictive distributions for $H_{S}$ and the maximum of $H_{S}$ corresponding to any return period of interest by simulation under the estimated model. For response $y^{*}$ of interest, with known density $f\left(y^{*} \mid \Omega\right)$ potentially restricted to a specified (joint) covariate interval, the posterior predictive density $f\left(y^{*} \mid \boldsymbol{y}, \boldsymbol{c}\right)$ of $y^{*}$ is $\int f\left(y^{*} \mid \Omega\right) f(\Omega \mid \boldsymbol{y}, \boldsymbol{c}) d \Omega$. Figure 6 illustrates model validation by comparison of estimates for the distribution of $H_{S}$ corresponding to the period of the original sample. The empirical estimate, found simply from sorting the original sample and shown as a solid red curve, is in good agreement with predictive simulations under the model, both omni-directionally and for 8 directional octants. Agreement is also good between the actual numbers of events observed in each directional sector examined and the corresponding median number of simulated events.

Figure 7 gives a directional-seasonal plot for the predictive distribution of the 100-year maximum. The left-hand panel shows directional and seasonal variability of the median estimate from multiple realisations of time-series of period 100 years under the directional-seasonal model. The right hand panel summarises seasonal variation of the predictive distribution for each of 8 directional octants. Again, there is systematic directional and seasonal variation present. For design purposes, extreme value inferences are usually reported in terms of directional and seasonal return value distributions, for return periods such as 100 years (Figure 8). Return value estimates such are these are critical for design and reliability assessments of marine and coastal structures. They indicate the need to consider directional and seasonal variability of extreme ocean environments to maximise safety and operability. They can also be used as a basis for decision making concerning the relative risk of different short-term marine operations.

\section{Discussion and conclusions}

We report the development of a simple piecewise model for a sample of peaks-over-threshold, non-stationary with respect to multidimensional covariates, estimated using Bayesian inference. We demonstrated the utility 

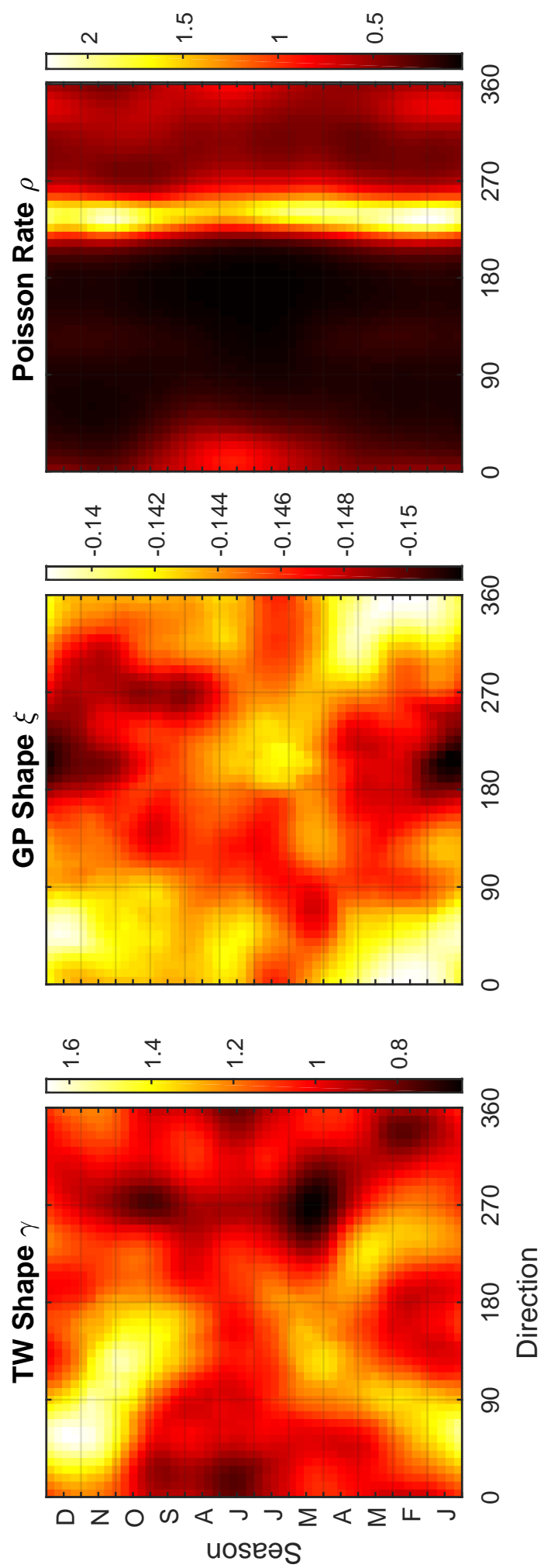
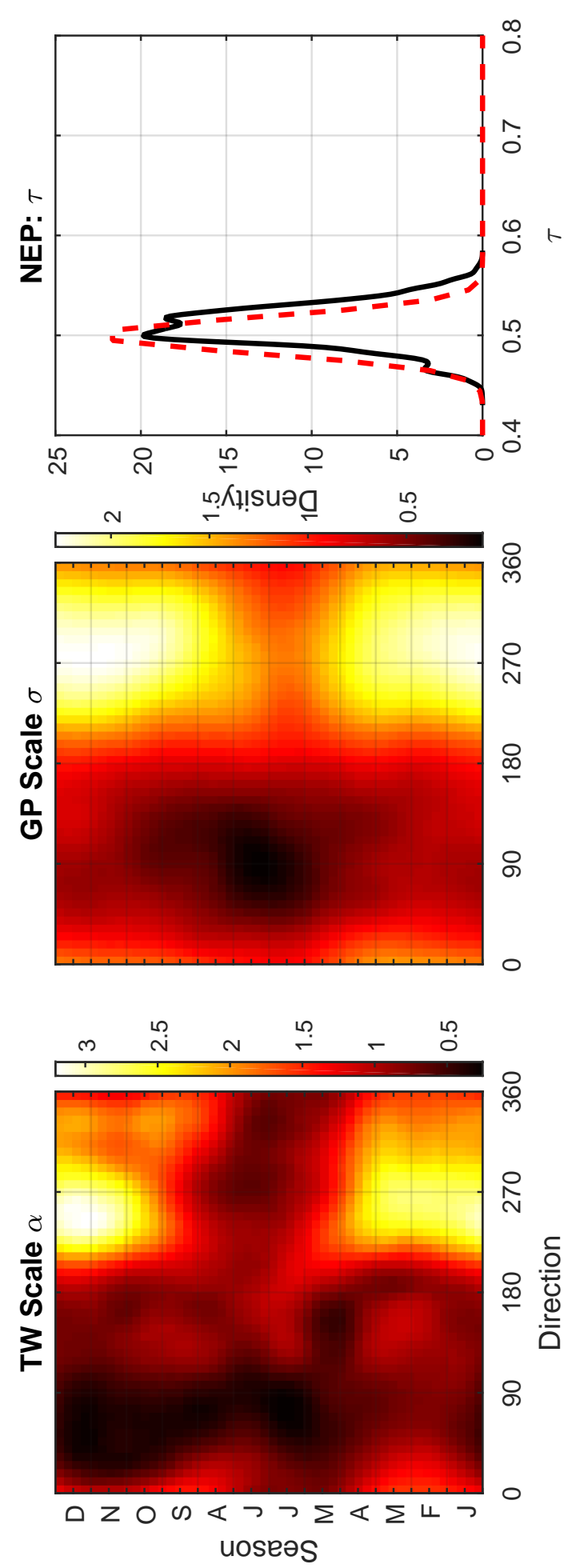

0
0
0
0
0
0

苟

离

๑

읏

0
0
0
0
0
0
0
0
0
0
0
0
0
0
0
a
0

의

$3 \frac{0}{5}$

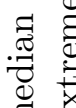

द्व

.0ี

式.

․

ธิ

응.

을

డే

चี

竞

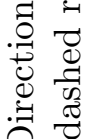

A

$\ddot{\forall} . \tilde{\sigma}$

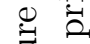

点 

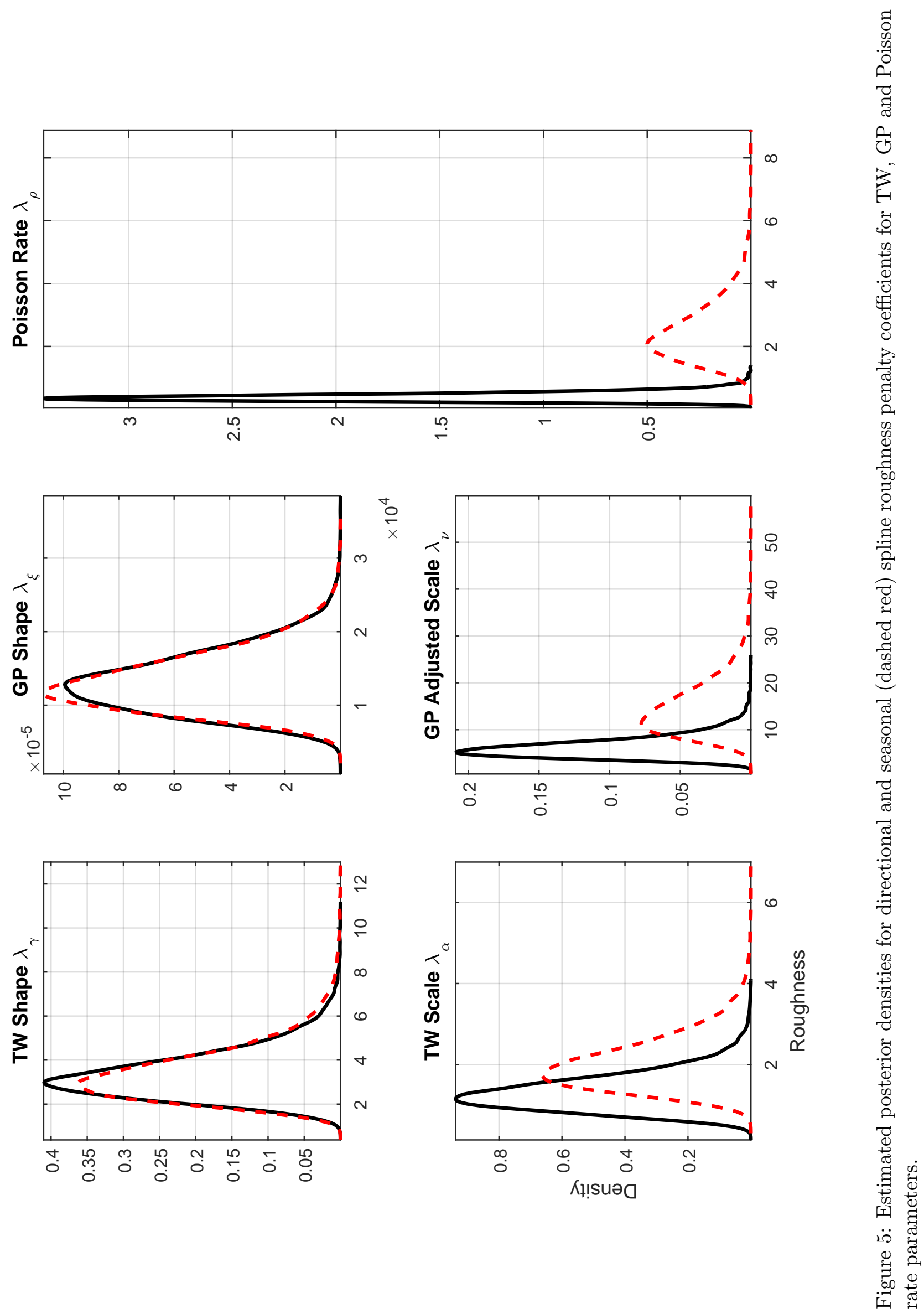

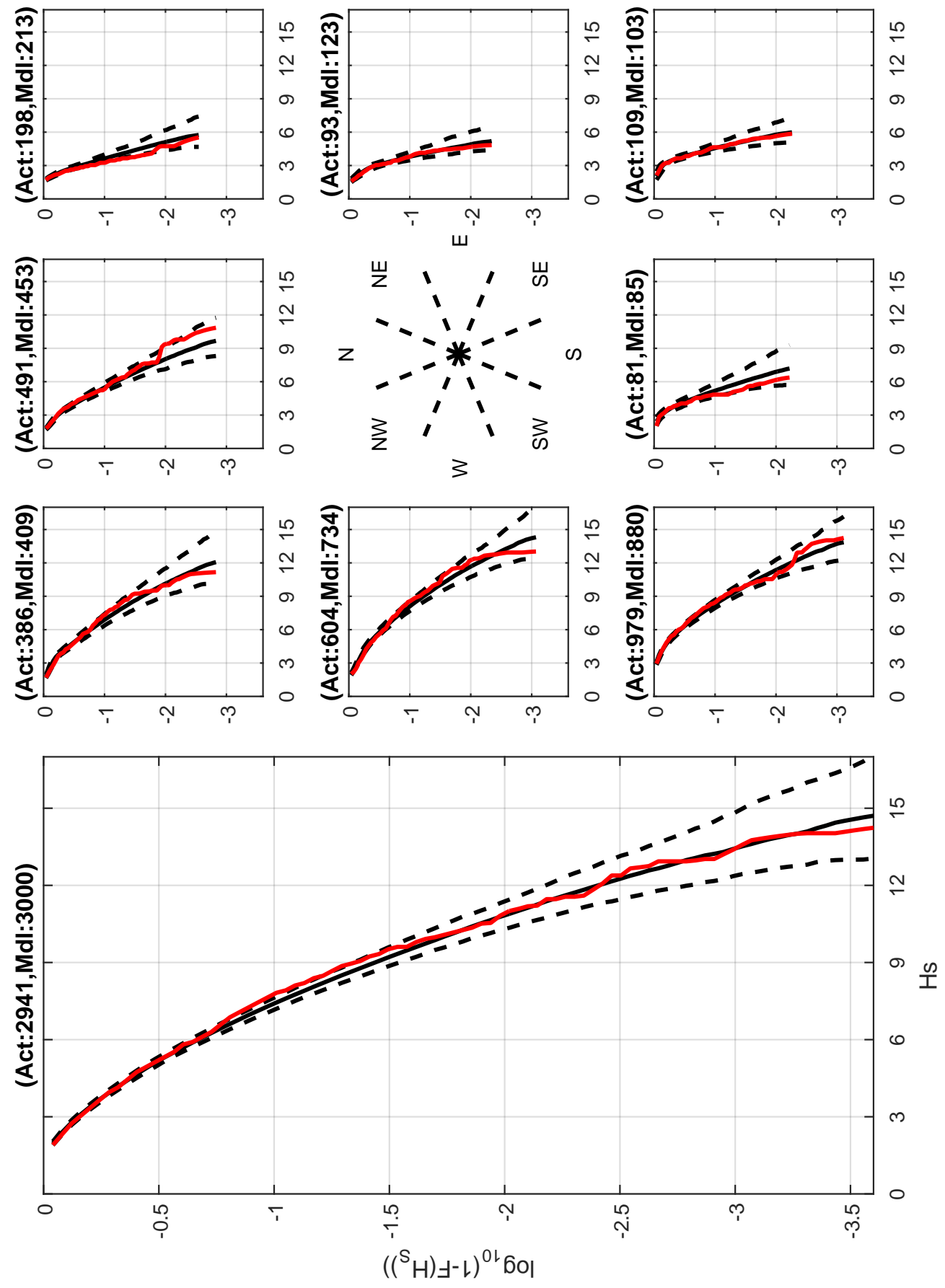

पे oै

을

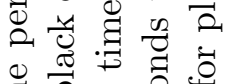

웜

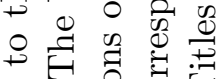

$\infty$ 更

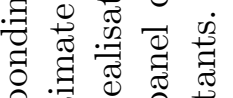

逢实

ฮี

क马

항 द्व

घี

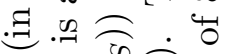

w

可政。

잉 1 व

若

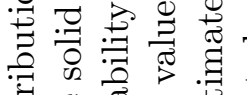

昰

븐

‡

苛热

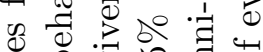

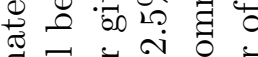

范

के 0 \%

$+$

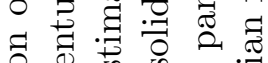

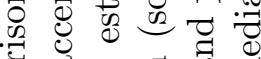

สี

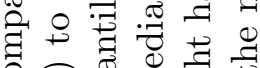

○ 2 ฮ

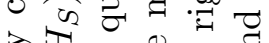
s.

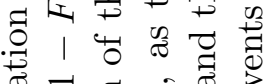

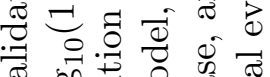

का

ब

过哥

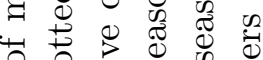

0 .

苛它记

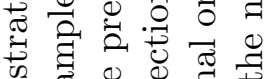

嗮

$\exists$ 匹 $\ddot{0} \cdot 0.0$ 记 总 

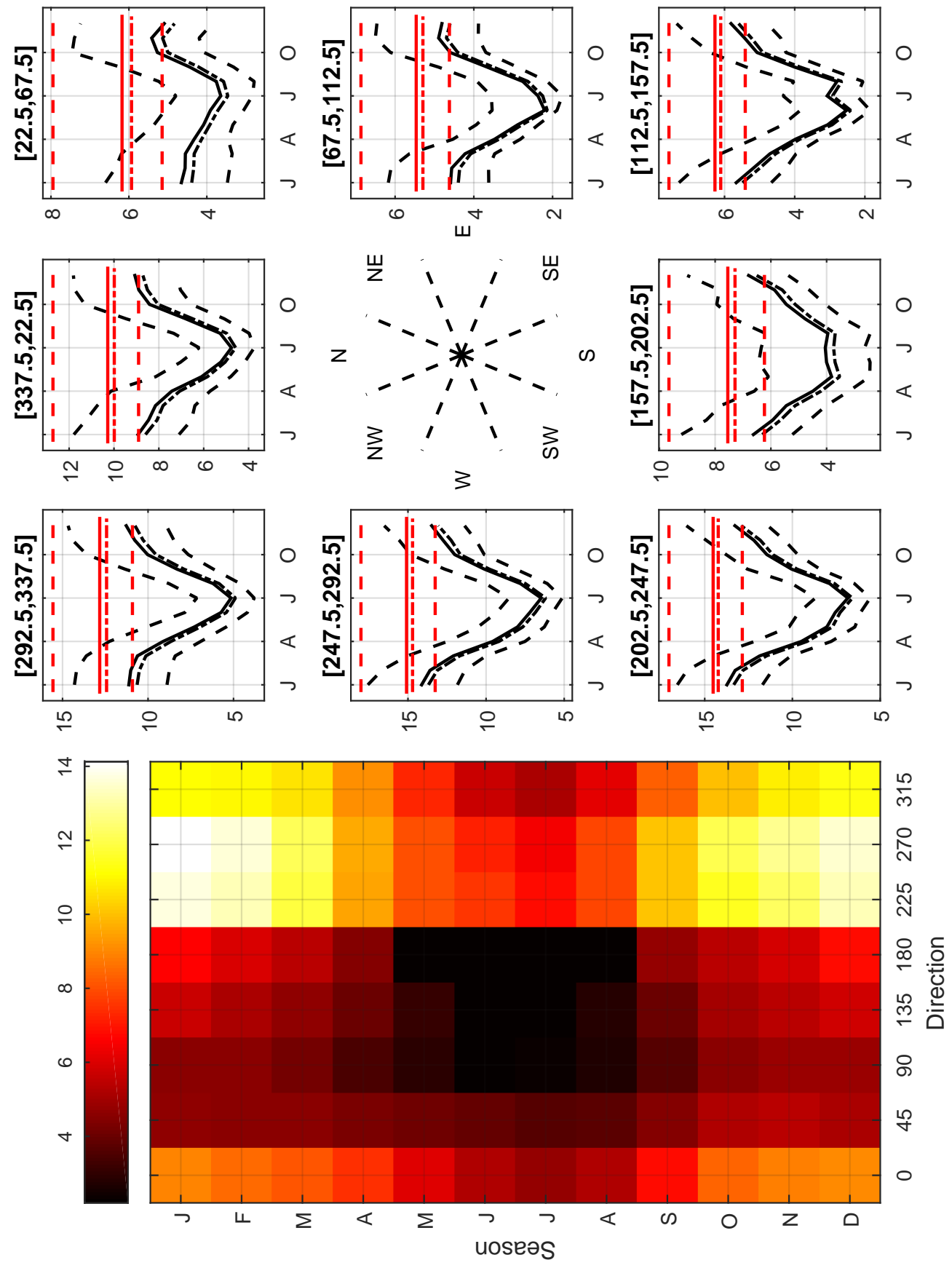

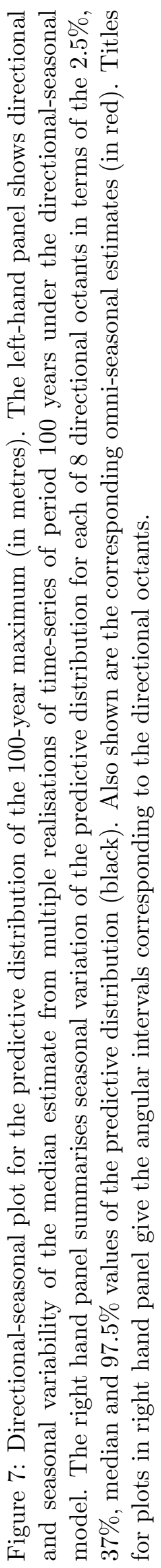



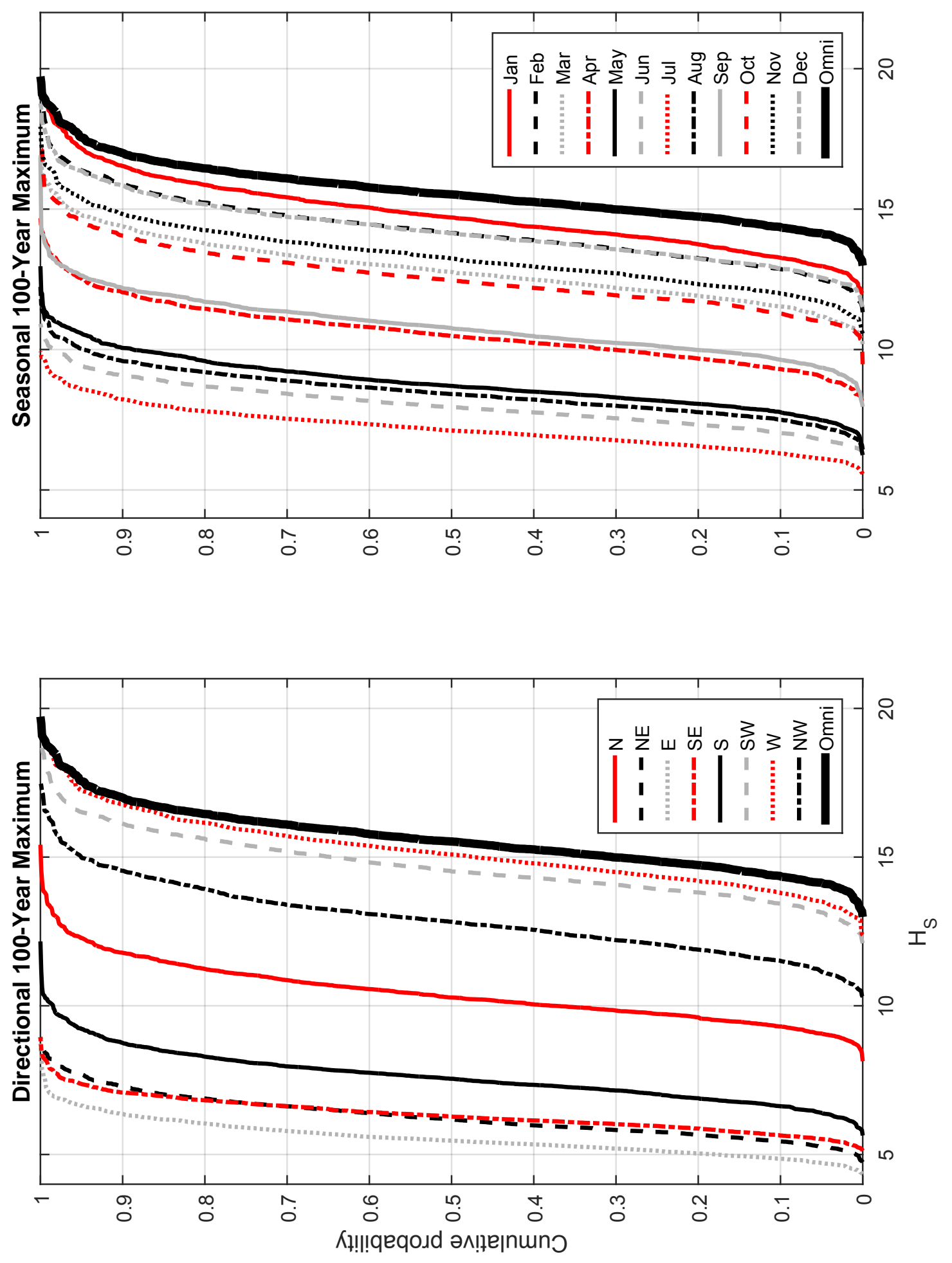
of the model in application to a directional-seasonal analysis of storm peak significant wave height at a northern North Sea location, and estimation of predictive directional-seasonal return value distributions necessary for the design and reliability assessment of marine and coastal structures.

These authors have performed a number of recent analyses similar to that reported here using maximum likelihood inference, incorporating cross-validation to estimate optimal spline roughness penalty coefficients and an all-encompassing bootstrap scheme to estimate uncertainties in model parameters and return values (e.g. Jonathan et al. 2014, Feld et al. 2015). In particular, we have performed a direct comparison of maximum likelihood and Bayesian inference for the South China Sea application (reported in Randell et al. 2015), obtaining good agreement for model parameter and return value estimates. Moreover, maximum likelihood inferences for the northern North Sea location considered here are provided in the Supplementary Material accompanying this work, again showing good agreement between maximum likelihood and Bayesian inferences. In comparison with these recent studies, the current Bayesian approach appears to offer a number of advantages. Firstly, inference is computationally considerably less demanding than maximum likelihood inference incorporating cross-validation and bootstrapping, particularly for estimation of spline roughness penalty coefficients. Model and return value estimation for a single MCMC chain can be completed in 30 minutes using modest computational resources (e.g. a single core PC with 2.7GHz processor and 4GB RAM, running MATLAB software in Microsoft Windows 7). Secondly, it appears that inference schemes which sample a function in a random but directed fashion, rather that seeking function minimum deterministically, provide more stable estimation for the models and samples considered. Finally, for the Bayesian scheme, estimation of different spline roughness penalty coefficients for each model parameter in each (marginal) covariate is straightforward; achieving the same within the maximum likelihood setting would be computationally more demanding. Numerous improvements and enhancements of the approach are currently under consideration. The choice of distributional form for the "body" part of the piecewise density somewhat arbitrary, although there is physical reasoning supporting the choice of Weibull distribution. In the current formulation, the piecewise density is discontinuous at the extreme value threshold $\psi$, although the cumulative distribution function is continuous on $\left(\zeta, y^{+}\right)$for given values of covariates. Estimation of spline roughness penalties $\left(\boldsymbol{\delta}_{\eta}\right.$ for $\left.\eta \in\{\rho, \alpha, \gamma, \nu, \xi\}\right)$ as in Brezger and Lang (2006) might be considered, although sampling from the prior performs well. Estimating $\boldsymbol{\delta}_{\eta}$ would come at some computational cost however because of the large number of penalties involved. From an applications perspective, current work is centred on evaluating the approach for higher-dimensional covariates (e.g. longitude, latitude, direction and season), enabling coherent (marginal) spatial analysis over large spatial domains (e.g. Raghupathi et al. 2016).

Additional information and supplementary material for this article is available online at the journals website.

\section{Acknowledgement}

We acknowledge discussions with Graham Feld, Matthew Jones and Rasmus Kulseng at Shell and Jonathan Tawn at Lancaster University. We are also grateful to two anonymous reviewers for thorough and thoughtful review of the work. The WAM hindcast is courtesy of Reistad et al. (2011). MATLAB algorithms for estimation of non-stationary extreme value models is available via Northrop et al. (2016). Further assistance is available from the authors on request.

\section{Appendix}

For the models described in Section 3 for event size and rate of occurrence, with parameter set $\Omega$, full conditional distributions to be sampled are

$$
\begin{aligned}
f(\tau \mid \boldsymbol{y}, \Omega \backslash \tau) & \propto f(\boldsymbol{y} \mid \tau, \Omega \backslash \tau) \times f(\tau) \\
f\left(\boldsymbol{\beta}_{\eta} \mid \boldsymbol{y}, \Omega \backslash \boldsymbol{\beta}_{\eta}\right) & \propto f\left(\boldsymbol{y} \mid \boldsymbol{\beta}_{\eta}, \Omega \backslash \boldsymbol{\beta}_{\eta}\right) \times f\left(\boldsymbol{\beta}_{\eta} \mid \boldsymbol{\delta}_{\eta}, \boldsymbol{\lambda}_{\eta}\right) \\
f\left(\boldsymbol{\lambda}_{\eta} \mid \boldsymbol{y}, \Omega \backslash \boldsymbol{\lambda}_{\eta}\right) & \propto f\left(\boldsymbol{\beta}_{\eta} \mid \boldsymbol{\delta}_{\eta}, \boldsymbol{\lambda}_{\eta}\right) \times f\left(\boldsymbol{\lambda}_{\eta}\right)
\end{aligned}
$$


where $\eta$ is each of $\rho, \alpha, \gamma, \nu$ and $\xi$ in turn, and the distribution of $\boldsymbol{\delta}_{\eta}$ is assumed known (Section 3.4). We generate a sample from the posterior distribution of $\Omega$ by sampling sequentially from the full conditional distributions for $\tau$, then the triplets of vectors $\left\{\boldsymbol{\beta}_{\eta}, \boldsymbol{\lambda}_{\eta}, \boldsymbol{\delta}_{\eta}\right\}$ for $\eta \in\{\rho, \alpha, \gamma, \xi, \nu\}$ in order, a large number of times. By construction, the full conditional for $\boldsymbol{\lambda}_{\eta}$ is available in closed form for all choices of $\eta$ (Brezger and Lang 2006), and hence Gibbs sampling is used. Sampling $\boldsymbol{\delta}_{\eta}$ from its (prior) distribution is also straightforward. Sampling from full conditionals for $\tau$ and $\boldsymbol{\beta}_{\eta}$ for $\eta \in\{\rho, \alpha, \gamma, \xi, \nu\}$ is achieved using MH in Gibbs. For any parameter (or vector of parameters) $\omega$, we accept transition from state $\omega$ to $\omega^{*}$ with probability

$$
\min \left(1, \frac{f\left(\omega^{*} \mid \Omega \backslash \omega^{*}\right) g\left(\omega^{*} \rightarrow \omega\right)}{f(\omega \mid \Omega \backslash \omega) g\left(\omega \rightarrow \omega^{*}\right)}\right)
$$

for proposal density $g$. For sampling of spline parameter vectors, correlated proposals are essential to reasonable chain mixing especially for larger problems. Proposals are generated using in one of two ways. For $\eta \in\{\alpha, \gamma\}$, random walk MH proposals $\boldsymbol{\beta}_{\eta}^{*}$ given current state $\boldsymbol{\beta}_{\eta}$ take the form $\boldsymbol{\beta}_{\eta}^{*}=\boldsymbol{\beta}_{\eta}+\left(\boldsymbol{B}^{\prime} \boldsymbol{B}+\boldsymbol{P}\right)^{-1} \boldsymbol{B}^{\prime} \boldsymbol{B}\left(\boldsymbol{B}^{\prime} \boldsymbol{B}+\boldsymbol{P}\right)^{-1} \kappa_{\eta} \epsilon$, where $\epsilon \sim N(0,1)$ and $\kappa_{\eta}$ is adjusted to regulate proposal acceptance rate. This form of proposal is motivated by consideration of smoothing splines or the ridge regression $\boldsymbol{y}=\boldsymbol{B} \boldsymbol{\beta}+\boldsymbol{\epsilon}$ where $\boldsymbol{\epsilon} \sim N(0, \boldsymbol{I})$. If $\boldsymbol{\beta}$ has normal prior $\boldsymbol{\beta} \sim N\left(\mathbf{0}, \boldsymbol{P}^{-1}\right)$, its posterior variance is $\left(\boldsymbol{B}^{\prime} \boldsymbol{B}+\boldsymbol{P}\right)^{-1} \boldsymbol{B}^{\prime} \boldsymbol{B}\left(\boldsymbol{B}^{\prime} \boldsymbol{B}+\boldsymbol{P}\right)^{-1}$. Proposals for $\tau$ are generated using a simple Gaussian random walk, again with variance adjustment on the fly to achieve suitable acceptance rate. Note that proposal densities for $\tau$ and $\boldsymbol{\beta}_{\eta}(\eta \in\{\alpha, \gamma\})$ are symmetric such that $g\left(\omega^{*} \rightarrow \omega\right)=g\left(\omega \rightarrow \omega^{*}\right)$, with resulting simplification of the expression for acceptance probability above. For $\eta \in\{\rho, \nu, \xi\}$, we propose $\boldsymbol{\beta}_{\eta}^{*}$ using derivatives of the target distribution at the current sample. This promotes proposals in regions of higher probability, at the additional computational cost of computing necessary derivatives and matrix inverses. Asymmetric random walk proposals are made as $\boldsymbol{\beta}_{\eta}^{*}=\boldsymbol{\beta}_{\eta}+\frac{1}{2} \kappa_{\eta}^{2} H^{-1} G+\kappa_{\eta} H^{-1 / 2} \epsilon$, where $H$ is the second derivative matrix of negative $\log$ full conditional for $\boldsymbol{\beta}_{\eta}$ with respect to $\boldsymbol{\beta}_{\eta}$ evaluated at the current state, and $\mathrm{G}$ is the corresponding gradient. The value of $\kappa_{\eta}$ is set to achieve suitable acceptance rate. We note the obvious similarity between mMALA and the back-fitting algorithm (e.g. Green and Silverman 1994, Davison 2003) for maximum likelihood estimation. As in the back-fitting algorithm, the constraint $\xi>-0.5$ is necessary to apply the mMALA algorithm in the generalised Pareto case. Further computational details and expressions for derivatives are given in Jones et al. (2016).

The table below gives prior distributional forms and parameter values used for $\tau$ and elements of $\delta$ and $\lambda$.

\begin{tabular}{|c|c|l|l|}
\hline Parameter & Distribution & $a$ & $b$ \\
\hline$\tau$ & $B(a, b)$ & 100 & 100 \\
$\delta$ & $\Gamma(a, b)$ & $\frac{1}{2}$ & $\frac{1}{2}$ \\
$\lambda$ & $\Gamma(a, b)$ & $10^{-3}$ & $10^{-3}$ \\
\hline
\end{tabular}

Prior distributions for $\tau$ and components of $\delta$ and $\lambda$.

We note that the prior for $\tau$ is relatively informative compared with those of other parameters, primarily to retain a sufficient proportion of the sample below extreme value threshold $\psi$ for reasonable confidence in predictions of return values, as discussed in te Supplementary Material.

\section{References}

Aarnes, O. J., Breivik, O., Reistad, M., 2012. Wave extremes in the Northeast Atlantic. J. Climate 25, 15291543.

Anderson, C., Carter, D., Cotton, P., 2001. Wave climate variability and impact on offshore design extremes. Report commissioned from the University of Sheffield and Satellite Observing Systems for Shell International.

Ashkenazy, Y., Gildor, H., 2011. On the Probability and Spatial Distribution of Ocean Surface Currents. Journal of Physical Oceanography 41 (12), 2295-2306. 
Battjes, J. A., Groenendijk, H. W., 2000. Wave height distributions on shallow foreshores. Coastal Eng. 40, $161-182$.

Behrens, C. N., Lopes, H. F., Gamerman, D., 2004. Bayesian analysis of extreme events with threshold estimation. Stat. Modelling 4, 227-244.

Beirlant, J., Goegebeur, Y., Segers, J., Teugels, J., 2004. Statistics of Extremes: theory and applications. Wiley.

Breivik, O., Aarnes, O. J., Bidlot, J.-R., Carrasco, A., Saetra, O., 2013. Wave extremes in the North East Atlantic from ensemble forecasts. J. Climate 26, 7525-7540.

Brezger, A., Lang, S., 2006. Generalized structured additive regression based on Bayesian P-splines. Comput. Statist. Data Anal. 50, 967-991.

Chavez-Demoulin, V., Davison, A., 2005. Generalized additive modelling of sample extremes. J. Roy. Statist. Soc. Series C: Applied Statistics 54, 207-222.

Cheng, L., Aghakouchak, A., Gilleland, E., Katz, R. W., 2014. Non-stationary extreme value analysis in a changing climate. Clim. Change, 353-369.

Chu, P. C., 2008. Probability distribution function of the upper equatorial pacific current speeds. Geophys. Res. Letters 35, 112606.

Coles, S., Walshaw, D., 1994. Directional modelling of extreme wind speeds. Applied Statistics 43, $139-157$.

Coles, S. G., Powell, E. A., 1996. Bayesian methods in extreme value modelling: a review and new developments. International Statistics Review 64, 119-136.

Coles, S. G., Tawn, J. A., 1996. A Bayesian analysis of extreme rainfall data. Applied Statistics 45, 463-478.

Coles, S. G., Tawn, J. A., 2005. Bayesian modelling of extreme sea surges on the UK east coast. Phil. Trans. R. Soc. A 363, 1387-1406.

Cooley, D., Naveau, P., Jomelli, V., Rabatel, A., Grancher, D., 2006. A Bayesian hierarchical extreme value model for lichenometry. Environmetrics 17, 555-574.

Currie, I. D., Durban, M., Eilers, P. H. C., 2006. Generalized linear array models with applications to multidimensional smoothing. J. Roy. Statist. Soc. B 68, 259-280.

Davison, A., Smith, R. L., 1990. Models for exceedances over high thresholds. J. R. Statist. Soc. B 52, 393.

Davison, A. C., 2003. Statistical models. Cambridge University Press.

Davison, A. C., Padoan, S. A., Ribatet, M., 2012. Statistical modelling of spatial extremes. Statist. Sci. 27, $161-186$.

Eilers, P. H. C., Currie, I. D., Durban, M., 2006. Fast and compact smoothing on multi-dimensional grids. Comput. Statist. Data Anal. 50, 61-76.

Ewans, K. C., Jonathan, P., 2008. The effect of directionality on northern North Sea extreme wave design criteria. J. Offshore. Arct. Eng. 130, 10.

Feld, G., Randell, D., Wu, Y., Ewans, K., Jonathan, P., 2015. Estimation of storm peak and intra-storm directional-seasonal design conditions in the North Sea. J. Offshore. Arct. Eng. 137, 021102:1-15.

Ferreira, J. A., Guedes Soares, C., 2000. Modelling distributions of significant wave height. Coastal Eng. 40 (4), $361-374$. 
Forristall, G. Z., 1978. On the statistical distribution of wave heights in a storm. J. Geophys. Res. 83, $2353-2358$.

Frigessi, A., Haug, O., Rue, H., 2002. A dynamic mixture model for unsupervised tail estimation without threshold selection. Extremes 5, 219-235.

Gamerman, D., Lopes, H. F., 2006. Markov chain Monte Carlo: stochastic simulation for Bayesian inference. Taylor \& Francis.

Girolami, M., Calderhead, B., 2011. Riemann manifold Langevin and Hamiltonian Monte Carlo methods. J. Roy. Statist. Soc. B 73, 123-214.

Green, P. J., Silverman, B., 1994. Nonparametric regression and generalised linear models: A roughness penalty approach. Chapman and Hall, London, UK.

Guedes-Soares, C., Scotto, M., 2001. Modelling uncertainty in long-term predictions of significant wave height. Ocean Eng. 28, 329.

Haver, S., 1985. Wave climate off northern Norway. Appl. Ocean Res. 7, 85-92.

Jonathan, P., Ewans, K. C., Forristall, G. Z., 2008. Statistical estimation of extreme ocean environments: The requirement for modelling directionality and other covariate effects. Ocean Eng. 35, 1211-1225.

Jonathan, P., Randell, D., Wu, Y., Ewans, K., 2014. Return level estimation from non-stationary spatial data exhibiting multidimensional covariate effects. Ocean Eng. 88, 520-532.

Jones, M., Randell, D., Ewans, K., Jonathan, P., 2016. Statistics of extreme ocean environments: non-stationary inference for directionality and other covariate effects. Ocean Eng. 119, 30-46.

MacDonald, A., Scarrott, C. J., Lee, D., Darlow, B., Reale, M., Russell, G., 2011. A flexible extreme value mixture model. Comput. Statist. Data Anal. 55, 2137-2157.

Marx, B. D., Eilers, P. H. C., 1998. Direct generalised additive modelling with penalised likelihood. Comput. Statist. Data Anal. 28, 193-209.

Mendes, J. M., de Zea Bermudez, P. C., Pereira, J., Turkman, K. F., Vasconcelos, M. J. P., 2010. Spatial extremes of wildfire sizes: Bayesian hierarchical models for extremes. Environ. Ecol. Stat. 17, 1-28.

Mendez, F. J., Menendez, M., Luceno, A., Medina, R., Graham, N. E., 2008. Seasonality and duration in extreme value distributions of significant wave height. Ocean Eng. 35, 131-138.

Monahan, A. H., 2006. The probability distribution of sea surface wind speeds. Part I: theory and seawinds observations. J. Climate 19, 497-520.

Morgan, E. C., Lackner, M., Vogel, R. M., Baise, L. G., 2011. Probability distributions for offshore wind speeds. Energ. Convers. Manage. 52 (1), 15-26.

Northrop, P., Attalides, N., Jonathan, P., 2015. Cross-validatory extreme value threshold selection and uncertainty with application to wave heights. (Submitted to J. Roy. Statist. Soc. C in April 2015, draft at www.lancs.ac.uk/ jonathan).

Northrop, P., Jonathan, P., Randell, D., 2016. Threshold modeling of nonstationary extremes. In: Dey, D., Yan, J. (Eds.), Extreme Value Modeling and Risk Analysis: Methods and Applications (to appear). Chapman and Hall / CRC, pp. 87-108.

Ortego Martinez, M. I., Egozcue Rubi, J. J., Tolosana Delgado, R., 2014. Bayesian trend analysis of extreme wind using observed and hindcast series off Catalan coast, NW Mediterranean Sea. Nat. Hazards Earth Syst. Sci. Discuss. 2, 799-824. 
Oumow, B., de Carvalho, M., Davison, A. C., 2012. Bayesian P-spline mixture modeling of extreme forest temperatures. Available at www.mat.puc.cl/ mdecarvalho.

Prevosto, M., Krogstad, H. E., Robin, A., 2000. Probability distributions for maximum wave and crest heights. Coastal Eng. 40, 329-360.

Raghupathi, L., Randell, D., Ewans, K., Jonathan, P., 2016. Fast computation of large scale marginal extremes with multi-dimensional covariates. Comput. Statist. Data Anal. 95, 243258.

Randell, D., Feld, G., Ewans, K., Jonathan, P., 2015. Distributions of return values for ocean wave characteristics in the South China Sea using directional-seasonal extreme value analysis. Environmetrics 26, 442-450.

Reistad, M., Breivik, O., Haakenstad, H., Aarnes, O. J., Furevik, B. R., Bidlot, J.-R., 2011. A high-resolution hindcast of wind and waves for the North sea, the Norwegian sea, and the Barents sea. J. Geophys. Res. 116, $1-18$.

Renard, B., Lang, M., Bois, P., 2006. Statistical analysis of extreme events in a nonstationary context via a Bayesian framework. Case study with peak-over-threshold data. Stoch. Env. Res. Risk A. 21, 97-112.

Scarrott, C., MacDonald, A., 2012. A review of extreme value threshold estimation and uncertainty quantification. Revstat 10, 33-60.

Scotto, M., Guedes-Soares, C., 2000. Modelling the long-term time series of significant wave height with nonlinear threshold models. Coastal Eng. 40, 313-327.

Scotto, M., Guedes-Soares, C., 2007. Bayesian inference for long-term prediction of significant wave height. Coastal Eng. 54, 393-400.

Smith, E. L., Walshaw, D., 2003. Modelling bivariate extremes in a region. In: In: Bayesian Statistics 7: Proceedings of the Seventh Valencia International Meeting, Dedicated to Dennis V. Lindley.

Smith, R. L., Naylor, J. C., 1987. A comparison of maximum likelihood and Bayesian estimators for the threeparameter Weibull distribution. J. Roy. Statist. Soc. C 36, 358-369.

Stephenson, A., 2016. Bayesian inference for extreme value modelling. In: Dey, D., Yan, J. (Eds.), Extreme Value Modeling and Risk Analysis: Methods and Applications (to appear). Chapman and Hall / CRC.

Ugarte, M. D., Goicoa, T., Etxeberria, J., Militino, A. F., 2012. Projections of cancer mortality risks using spatio-temporal P-spline models. Stat. Methods in Med. Res 21, 545-560.

Xifara, T., Sherlock, C., Livingstone, S., Byrne, S., Girolami, M., 2014. Langevin diffusions and the Metropolisadjusted Langevin algorithm. Stat. Probabil. Lett. 91 (2002), 14-19. 


\title{
Supplementary Material
}

\author{
Bayesian inference for non-stationary marginal extremes \\ by \\ D. Randell, K. Turnbull, K. Ewans and P. Jonathan
}

Please address all correspondence to philip.jonathan@shell.com or p.jonathan@lancaster.ac.uk

\section{Maximum likelihood inference}

It is interesting to compare Bayesian inference under the truncated Weibull - generalised Pareto model of the main text (henceforth here "the Bayesian model" for brevity) with maximum likelihood inference made using the approach described in Randell et al. [2015] (henceforth "the maximum likelihood model"), for the northern North Sea location considered in the main text. Here, we provide a brief discussion and illustration of a maximum likelihood inference, presenting figures equivalent to Figures 6-8 of the main text to facilitate direct comparison.

Both the Bayesian and maximum likelihood models make use of a generalised Pareto distribution, the parameters of which are non-stationary with respect to covariates, to describe the characteristics of extreme values. However, there are a number of differences between the approaches which might influence inferences. For instance, in the maximum likelihood model, a non-stationary extreme value threshold is estimated using quantile regression for a pre-specified non-exceedance threshold probability, as opposed to being estimated alongside other model parameters in a single inference. Further, in the maximum likelihood model, only one global roughness penalty coefficient $\lambda_{\eta}^{*}$ is estimated per model parameter $\eta$ (in the notation of the main text, despite the fact that directional and seasonal covariates may require different degrees of regularisation). To overcome this limitation, the relative weighting $w_{\eta 1}, w_{\eta 2}$ of directional and seasonal roughness penalty coefficients $\lambda_{\eta 1}, \lambda_{\eta 2}$ with respect to $\lambda_{\eta}^{*}$, such that $\lambda_{\eta 1}=w_{\eta 1} \lambda_{\eta}^{*}, \lambda_{\eta 2}=w_{\eta 2} \lambda_{\eta}^{*}$ is estimated prior to model estimation. We anticipate that the Bayesian model might provide more consistent inferences therefore when prior estimation of weights is difficult, as in the current application where seasonal variation of extreme value characteristics is more gradual than directional variation (see e.g. Figure 1 and Figure 5 of main text). We also make use of different "peak-picking" algorithms for the Bayesian and maximum likelihood models, so that the sets of storm peaks selected for analysis may show small differences in the number of storm peaks with storm peak values near the peak-picking threshold. This is immaterial for characterisation of extreme values.

Critical inferences from the extreme value models are return value distributions. We therefore focus the comparison on these.

Figure S1 (c.f. Figure 6 of the main text) illustrates model validation for the maximum likelihood model by comparison of estimates for the distribution of $H_{S}$ corresponding to the period of the original sample. The empirical estimate, found simply from sorting the original sample and shown as a solid red curve, is in good agreement with realisations under the model, both omni-directionally and for 8 directional octants. Agreement is also good between the actual numbers of events observed in each directional sector examined and the corresponding median number of simulated events. Moreover, Figure S1 and Figure 6 (main text) are in good agreement for the omni-directional omni-seasonal case, and the 8 directional octants.

Figure S2 (c.f. Figure 7 of the main text) gives a directional-seasonal plot for the distribution of the 100-year maximum for the maximum likelihood model. The left-hand panel shows directional and seasonal variability of the median estimate from multiple realisations of time-series of period 100 years under the directional-seasonal model. The right hand panel summarises seasonal variation for each of 8 directional octants. Again, there is systematic directional and seasonal variation present. Figure S2 is in good agreement with Figure 7 of the main text. In the eastern and southern octants, it appears that the Bayesian model has better identified the seasonal variation of the 100-year maximum, although there is evidence for seasonal variability in the estimated uncertainty band under the maximum likelihood model. We attribute this different to the ability to estimate relative direction and seasonal smoothness directly in the Bayesian inference, as discussed above.

For design purposes, as discussed in the main text, extreme value inferences are usually reported in terms of directional and seasonal return value distributions, for return periods such as 100 years, illustrated in Figure S3 for the model estimated using maximum likelihood. Again there is good agreement between Figure S3 and Figure 8 of the main text. First we note that the omni-directional omni-seasonal estimates are within $0.2 \mathrm{~m}$ of each other for any probability level between 0.25 and 0.75 . Next we note that the magnitudes and ordering of return value distributions for directional octants (left hand side) and calendar 
months (right hand side) are almost identical also. In particular, the most severe directional sectors in both analyses are western, south-western, followed by north-eastern, northern, southern in that order. The most severe monthly extremes are January, February and December (the latter two very similar), followed by November, March, October in that order in both analyses.

We conclude that inferences from the Bayesian model described in the main text, and the maximum likelihood model described in Randell et al. [2015] are comparable.

\section{Non-exceedance probability $\tau$}

For samples of reasonable size, generated under the truncated Weibull - generalised Pareto with pre-specified parameters, we are able to estimate all model parameters reasonably and routinely including extreme value threshold non-exceedance probability $\tau$ in general using the model described in the main text.

In our experience of maximum likelihood estimation of (potentially non-stationary) generalised Pareto models of storm peak significant wave height, specification of extreme value threshold is generally problematic. Standard diagnostics (e.g. mean excess plot, variation of estimated generalised Pareto shape parameter with $\tau$ ) often indicate that any of a number of different values of $\tau$ is plausible. For instance, Figure S6 in the Supplementary Material of Randell et al. [2015] explores the sensitivity of the omni-directional omni-seasonal 100-year return value of storm peak significant wave height from a directional-seasonal model to choices of $\tau \in[0.3,0.95]$. It is clear from the figure that return values are relatively insensitive to the choice of $\tau$ made. Further, the precision with which generalised Pareto model parameters are estimated generally improves with increasing sample size. The quality of inferences made (e.g. of return values) using the estimated generalised Pareto parameters also improves in general with sample size. It is therefore natural to select a value or range of values for $\tau$ as low as possible given acceptable extreme value model fit to the tail of the sample.

For the sample of northern North Sea storm peak significant wave height considered in the main text with the Bayesian truncated Weibull - generalised Pareto model, estimation of $\tau$ is equally challenging. We find that the sample is relatively uninformative about $\tau$, as illustrated in Figure S4. The figure summarises the posterior distribution of sample negative log likelihood, estimated using Bayesian inference for a sequence of different pre-specified values for $\tau$. There is some evidence that smaller values of $\tau$ provide somewhat better fit, and the suggestion of some preference for $\tau$ in the region of 0.85 . We have examined a number of different choices for the prior beta distribution of $\tau$; inferences in each case are consistent with the evidence from Figure S4. In specifying the choice of a prior B(100,100) distribution used in the main text, we attempted to balance the need for sufficient sample to be able to estimate the generalised Pareto tail parameters reasonably (i.e. $\tau$ relatively small), with keeping $\tau$ sufficiently large that fitting an extreme value model to the tail of the data is also reasonable, in the light of common practice of specifying a (fixed) conditional median threshold.

\section{References}

D. Randell, G. Feld, K. Ewans, and P. Jonathan. Distributions of return values for ocean wave characteristics in the South China Sea using directional-seasonal extreme value analysis. Environmetrics, 26:442-450, 2015. 

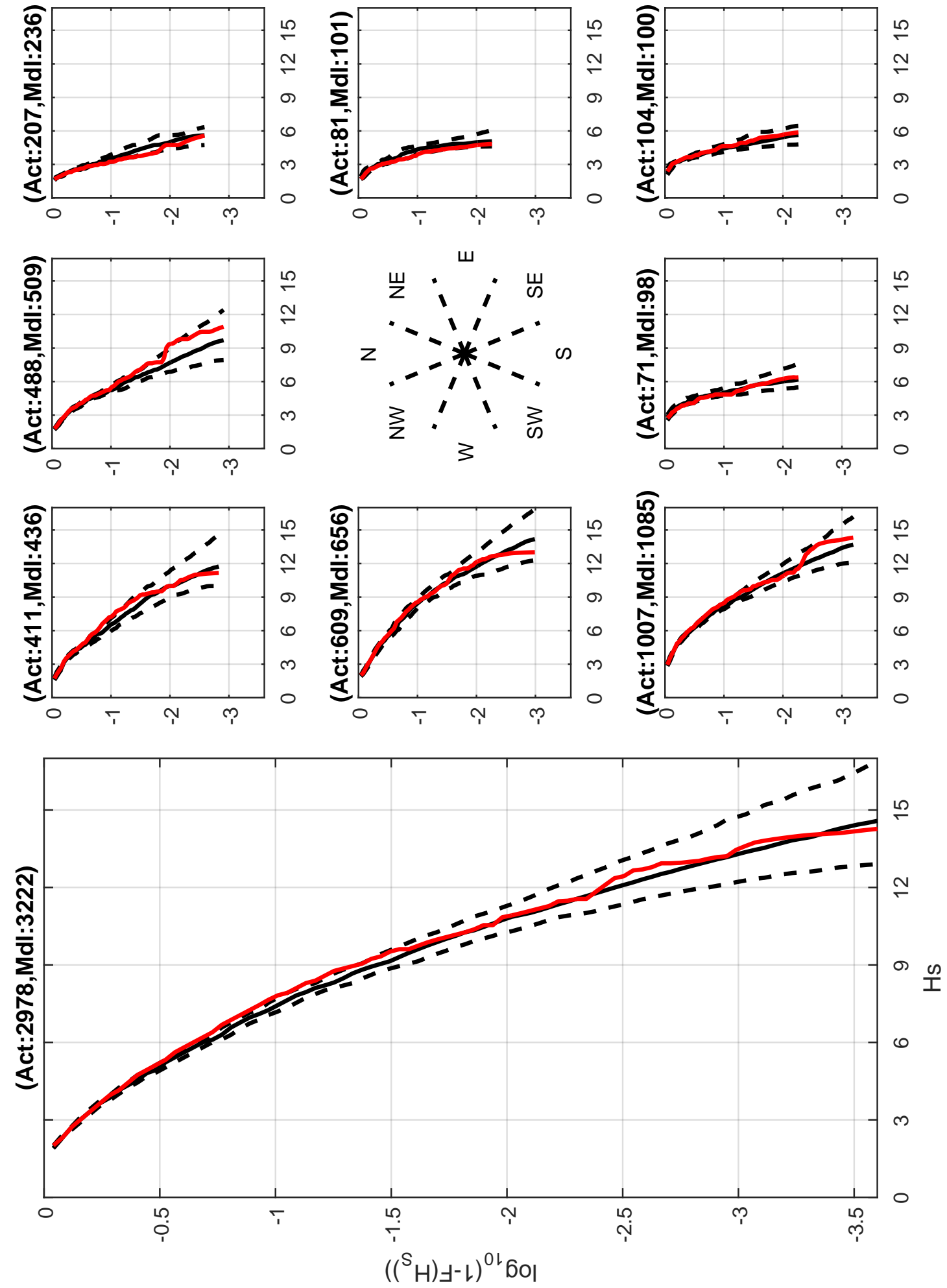

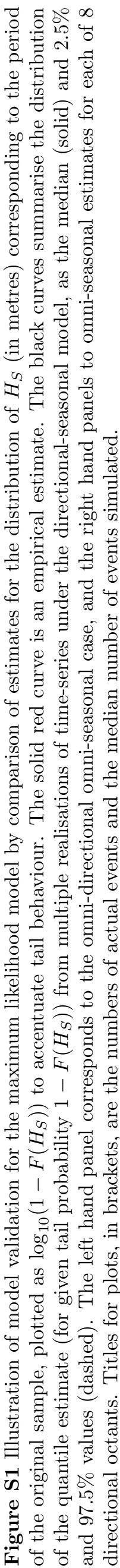



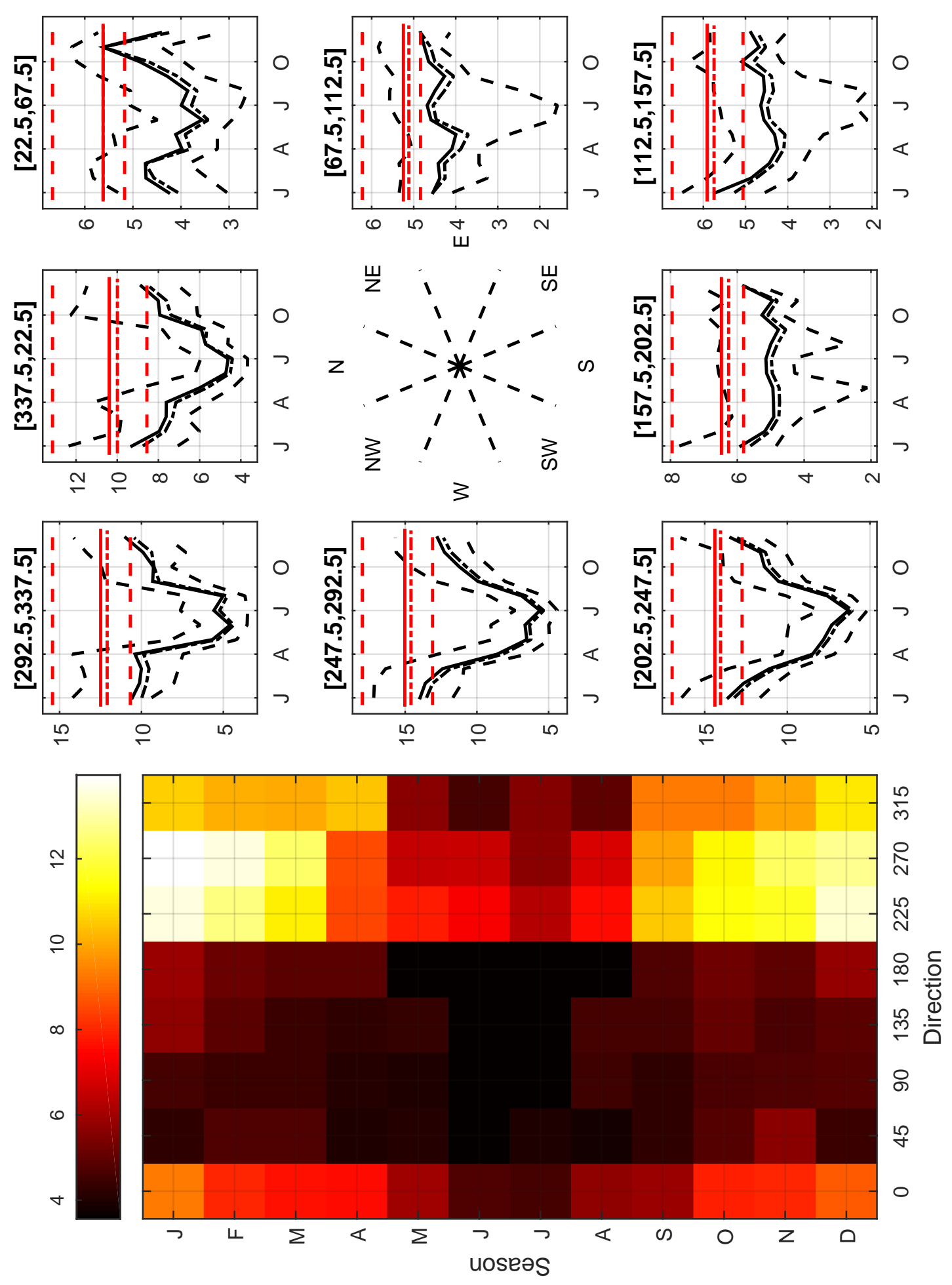

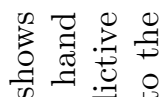

के

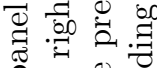

要类

चै

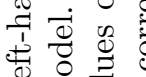

(

四沓

-

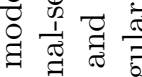

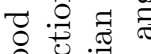

雪总

吾

园苛

क

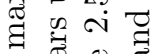

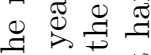

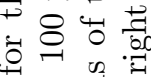

क्षे

要

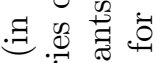

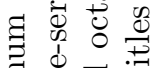

畓急

㟧

๘ี

今.

엄

$\Psi$ व

○. 式

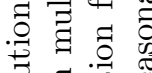

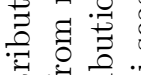

氙语寻

(])

.

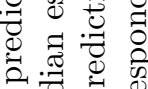

ठ

ఫ

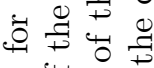

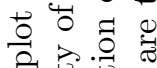

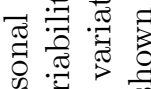

ठิ

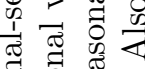

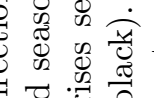

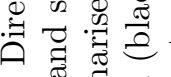

ฟ

के

0
0
0 

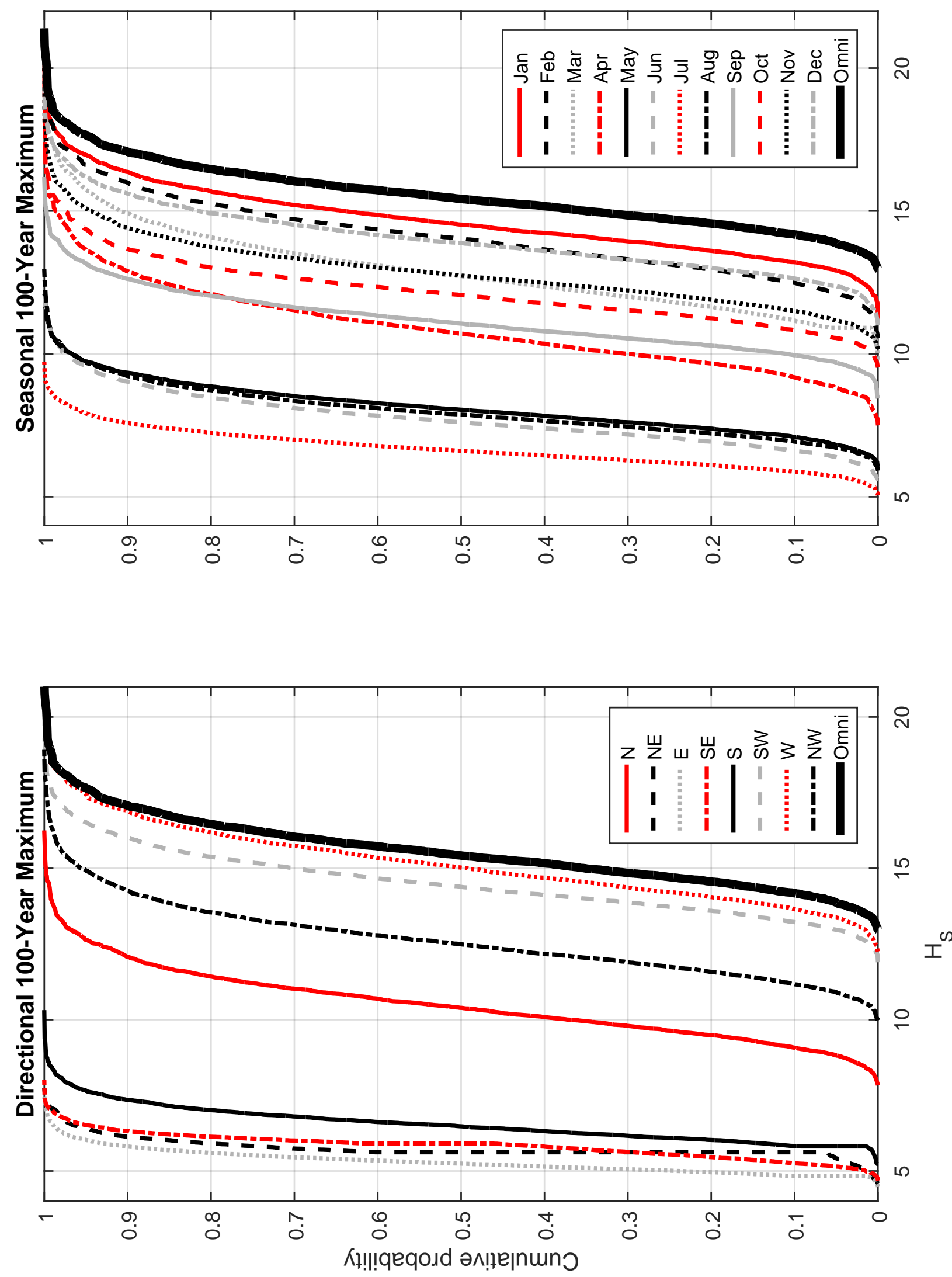


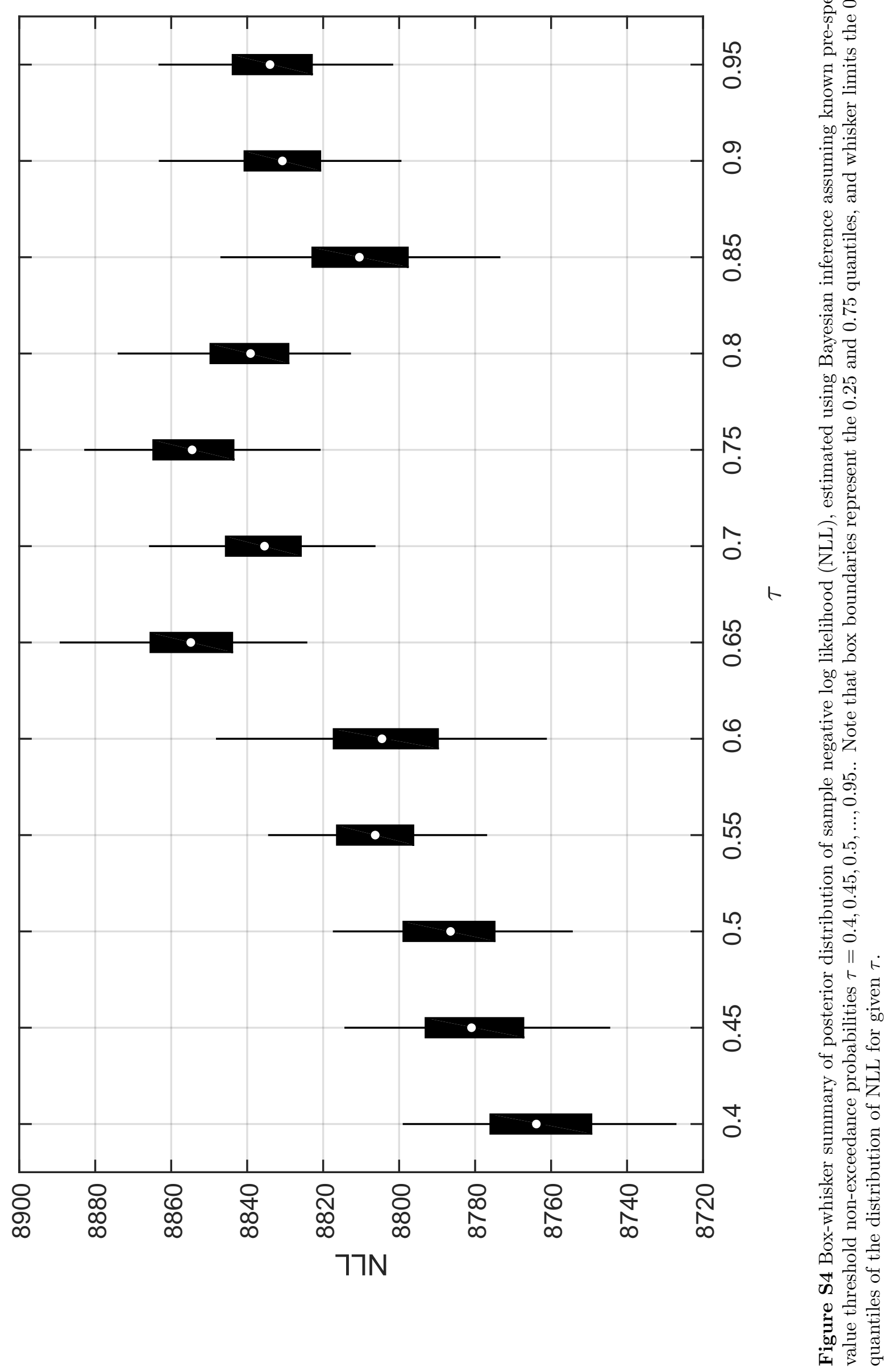

